\title{
Uptake of Oleate from Albumin Solutions by Rat Liver \\ Failure to Detect Catalysis of the Dissociation of Oleate from Albumin by an Albumin Receptor
}

Richard A. Weisiger and Wei-Lan Ma

Department of Medicine and the Liver Center, University of California, San Francisco, California 94143

\begin{abstract}
The hepatic removal of albumin-bound substances from plasma requires that they dissociate from albumin. Using indirect methods, we and others have proposed that dissociation may be catalyzed by interaction of albumin with the liver cell surface. This study looked for direct evidence of catalysis by comparing the rate of dissociation of oleate from albumin in vitro with the rate observed within the sinusoids of perfused rat liver. No evidence for catalysis was found. The rate of hepatic oleate removal from dilute albumin solutions did not exceed but instead closely paralleled the rate predicted from the in vitro dissociation rate constant $\left(0.14 \mathrm{~s}^{-1}\right)$. These results suggest that under some conditions the liver can remove unbound material from the sinusoids faster than it can be replenished by dissociation from albumin, resulting in dissociation-limited removal. However, dissociation of oleate does not appear to be catalyzed by the liver.
\end{abstract}

\section{Introduction}

Recent studies of the hepatic removal of albumin-bound substances have led to a reevaluation of traditional concepts regarding this process. The rate of transport, long thought to be determined solely by the unbound, or free, concentration in plasma, has been reported to correlate better with the albuminbound concentration in a variety of experimental models (1-7). A specific kinetic pattern has been reported that includes saturation of ligand removal as the concentration of albumin-ligand complexes is increased and competitive inhibition of removal by added albumin. To account for these and related data, it has been proposed that uptake may occur not only from the free ligand pool but also, though less efficiently, from the bound ligand pool by a mechanism involving transient binding of the albumin to a limited number of sites on the liver cell surface (1, $4,8)$. According to this albumin receptor model, the latter pathway predominates for extensively bound ligands such as fatty acids due to their very small free concentrations in plasma.

Although this model can account for the original kinetic observations (1) and has enjoyed some success in predicting subsequent findings (9), an alternative model has recently been

This work was presented at the Annual Meeting of the American Association for the Study of Liver Diseases, Chicago, IL, November 1985, and has previously appeared in abstract form (1985. Hepatology. 5:1012.)

Address correspondence to Richard A. Weisiger, M.D., Ph.D., 1120 HSW, University of California, San Francisco, CA 94143.

Received for publication 7 April 1986 and in revised form 24 November 1986.

J. Clin. Invest.

(c) The American Society for Clinical Investigation, Inc. 0021-9738/87/04/1070/08 \$1.00

Volume 79, April 1987, 1070-1077 proposed that does not require postulating an interaction of albumin with the liver cell (10). This dissociation-limited model retains the traditional assumption that the hepatic removal mechanism acts only on free ligand. It attributes the failure of the removal rate to reflect the equilibrium free ligand concentration to a lack of binding equilibrium within the hepatic sinusoids. This condition results when free ligand is removed by the liver faster than it can be replenished by dissociation from albumin. Because in this case dissociation of the bound ligand from albumin is the rate-limiting step in ligand transport, the apparent dependence of the removal rate on bound ligand is explained. This model has been shown to be fully consistent with the kinetic behavior previously attributed to an albumin receptor (10). The finding of albumin receptor kinetics in the perfused elasmobranch (skate) liver further supports this model, because these primitive vertebrates lack serum albumin and thus have no apparent reason to have evolved an albumin receptor (11). However, no comparison of these models in mammalian liver has been reported.

Discrimination has proven difficult because the albuminreceptor and dissociation-limited models predict virtually the same kinetic pattern. However, these models differ in the absolute rates of ligand removal that they predict. The dissociation-limited model predicts that removal of bound ligand cannot exceed the rate of spontaneous dissociation of ligand from albumin within the hepatic sinusoids, and that under specific dissociation-limited conditions the rate of removal will equal this rate $(10,11)$. In contrast, the albumin receptor model holds that dissociation is catalyzed by interaction of albumin with the cell surface $(1,4$, 8). The removal rate predicted by this model is thus not limited by the rate of spontaneous dissociation and may be much faster if catalysis is efficient.

The current study compares the rate of oleate removal from dilute albumin solutions by the perfused rat liver with the rate expected if uptake were limited by spontaneous dissociation of oleate from albumin within the hepatic sinusoids. We reasoned that a removal rate equal to the spontaneous dissociation rate would support the dissociation-limited model, whereas a more rapid rate would support catalysis of dissociation by the liver such as is predicted by the albumin receptor model.

\section{Methods}

Cyanogen bromide, sodium oleate, and bovine serum albumin (fraction V, essentially fatty acid free) were obtained from Sigma Chemical Co., St. Louis, MO. Lipidex 1000 was from Packard Instrument Co., Inc., Downers Grove, IL; agarose (Sepharose 4B) and blue dextran 2000 were from Pharmacia, Inc., Uppsala, Sweden. Albumin was covalently coupled to the agarose beads using cyanogen bromide as described by March and coworkers (12). Albumin was radioiodinated where specified by the chloramine $t$ method (13) to a level of not more than one iodine per molecule. All chemicals and solvents were of the highest commercially available grade. 
Solution preparation. All solutions were prepared with modified Krebs Henseleit buffers and were used within $4 \mathrm{~h}$ unless otherwise specified. Liver perfusion solutions were prepared as previously described (1) and equilibrated with $95 \%$ oxygen/5\% carbon dioxide before use.

For in vitro studies, $\left[9,10^{-3} \mathrm{H}\right]$ oleate in ethanol $\left(2-10 \mathrm{Ci} \cdot \mathrm{mmol}^{-1}\right.$, New England Nuclear, Boston, MA) was dried under nitrogen, redissolved in hexane, and stored over $10 \mathrm{vol}$ of water at $4^{\circ} \mathrm{C}$ until use. On the morning of the study an aliquot was dried and redissolved in $10 \mu \mathrm{l}$ propylene glycol before mixing with $1 \mathrm{ml}$ buffer containing the specified concentration of albumin. This buffer was the same as was used in liver perfusion except that $10 \mathrm{mM}$ tricine base (adjusted to $\mathrm{pH} 7.4$ at $23^{\circ} \mathrm{C}$ with $\mathrm{HCl}$ ) replaced bicarbonate and $0.02 \%$ sodium azide was added. Calcium and magnesium were replaced by sodium in the albumin-agarose but not the Lipidex experiments.

Liver perfusion. Perfusion was carried out as previously described $(1,14)$. Briefly, 7-9-g livers removed from 55-65-d-old fed male Sprague Dawley rats under ether anesthesia were perfused at $22-26 \mathrm{ml} \cdot \mathrm{min}^{-1}$ with a recirculating fluorocarbon emulsion (Oxypherol FC-43; Alpha Therapeutics, Los Angeles, CA) for $30 \mathrm{~min}$. The liver was then perfused single-pass for $4 \mathrm{~min}$ with $26-30 \mathrm{ml} \cdot \mathrm{min}^{-1} \mathrm{Krebs}$ Henseleit bicarbonate buffer to wash out the fluorocarbon and then with the same buffer plus $10 \mu \mathrm{M}$ albumin and $1 \mu \mathrm{M}\left[{ }^{3} \mathrm{H}\right]$ oleate for determination of oleate extraction. In all cases steady-state uptake was achieved within $60 \mathrm{~s}$, after which effluent samples were taken. Fatty acids were separated from radiolabeled metabolites by the method of Dole (15) before quantification of radioactivity by liquid scintillation counting.

Where specified, liver temperature was varied by passing the perfusate through a heat exchanger coil ( $40 \mathrm{~cm}$ of $2-\mathrm{mm}$ stainless steel tubing) before it entered the liver. Coil temperature was regulated using a recirculating water bath (Lauda K-2/R, FRG). Liver temperature was monitored with a remote probe capable of $0.1^{\circ} \mathrm{C}$ precision. During steadystate oleate uptake the coil was suddenly cooled with $4^{\circ} \mathrm{C}$ water, causing liver temperature to fall to $12-14^{\circ} \mathrm{C}$ and the oleate uptake rate to fall by $>80 \%$ within $\sim 60 \mathrm{~s}$. Oleate extraction was then measured at intervals as the temperature of the water bath was increased $\left(2^{\circ} \mathrm{C} \mathrm{min}{ }^{-1}\right)$. The temperature of the different liver lobes was typically uniform to within $1^{\circ} \mathrm{C}$. Perfusate $\mathrm{pH}$ varied by $<0.1 \mathrm{U}$ over the temperature range studied.

Hepatic viability in this system has been demonstrated by bile flow, perfusion pressure, oxygen consumption, and light and electron microscopy (14). Viability following perfusion at reduced temperature was assessed by comparing the initial rate of oleate removal at $37^{\circ} \mathrm{C}$ with that measured after cooling and rewarming of the liver. Only experiments in which the repeat determination was within $10 \%$ of the initial removal rate were further analyzed.

In vitro dissociation. In vitro dissociation rates were determined from the rate of transfer of oleate from albumin to acceptor beads capable of binding free oleate but not the oleate-albumin complexes (Fig. 1). When a sufficient concentration of acceptor is used, newly dissociated oleate binds to the acceptor beads before it can rebind to the albumin. Under these conditions, the rate of transfer of the oleate to the acceptor beads is determined by its rate of dissociation from albumin, allowing convenient measurement of the dissociation rate constant.

This approach has two potential weaknesses. First, dissociation might be nonspecifically catalyzed by a direct interaction of the albumin complexes with the acceptor beads. To evaluate this possibility, two different oleate acceptors were used. Second, errors could be introduced if transfer processes other than dissociation, such as diffusion across unstirred layers adjacent to the acceptor beads, significantly limited the transfer rate. To evaluate this possibility, two entirely different in vitro protocols were used.

Albumin-agarose method. The first method was modified from Svenson and coworkers (16). The oleate acceptor is bovine albumin covalently linked to agarose beads and transfer is assessed by rapid filtration. A suspension of albumin-agarose ( $17 \mathrm{ml}, 30-40 \%$ wet gel by volume) in Krebs tricine buffer was vigorously stirred in a small thermoregulated beaker. Typically, $100 \mu \mathrm{l}$ of $\left[{ }^{3} \mathrm{H}\right]$ oleate-albumin in the same buffer (molar ratio, 1:2; albumin concentration, $10 \mu \mathrm{M}$ ) was then added and $\sim 1 \mathrm{ml}$ of the resulting mixture removed at $5 \mathrm{~s}$ intervals. Samples were imme-

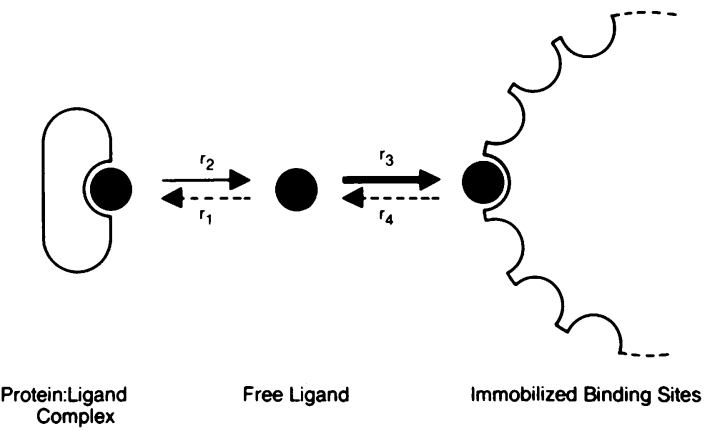

Figure 1. General method for measuring dissociation in vitro. Proteinligand complex is added to an excess of immobilized ligand-binding sites, and the net rate of ligand transfer to the acceptor is determined. For a sufficient concentration of acceptor, the rate of transfer will reflect the rate of dissociation of the ligand from the binding protein. $r_{2}$, first order dissociation rate constant $\left(\mathrm{s}^{-1}\right) ; r_{1}$, second order association rate constant $\left(\mathrm{M}^{-1} \cdot \mathrm{s}^{-1}\right) ; r_{3}$, pseudo-first order rate constant for binding of ligand to a given concentration of acceptor $\left(\mathrm{s}^{-1}\right) ; r_{4}$, first order rate constant for dissociation of the ligand from the acceptor $\left(\mathrm{s}^{-1}\right)$.

diately filtered through 24-mm glass fiber filters (GF/C; Whatman Ltd., England) using a 10-position vacuum manifold (FH224; Hoefer Scientific Instruments, San Francisco, CA). Aliquots of the filtrate were assayed for radioactivity by liquid scintillation counting. An unfiltered sample of the total suspension was also counted to allow determination of total radioactivity. Results were expressed as the fraction of the total oleate remaining in the soluble phase versus time. Control experiments indicated no measurable binding of oleate to the glassware or filters when albumin was present. In control studies, some binding of ${ }^{125} \mathrm{I}$-albumin to the albumin-agarose was detected. This was prevented by adding an inert carrier protein $(0.1 \%$ ovalbumin) to the gel suspension. Control studies indicated that ${ }^{125} \mathrm{I}$-albumin was fully equilibrated with the interior of the agarose beads within $5 \mathrm{~s}$ after mixing.

Lipidex method. The second method exploits the fact that oleate elutes down a column of acceptor beads only while bound to albumin. Dissociation leads to rapid and irreversible binding of the fatty acid to the column bed. If the time required for albumin to transit the column is known, the distribution of oleate along the length of the column following elution of the complex can be used to determine the dissociation rate constant (Fig. 2). The acceptor used in the current study was Lipidex, a hydrophobic reverse-phase exchanger that permits high flow rates. Although Lipidex binds fatty acids avidly, it does not bind albumin (17).

The theoretical basis for this approach was first developed by Hillier (18). The method is useful only when the rate constant for binding of free ligand to the acceptor is much greater than the rate constant for

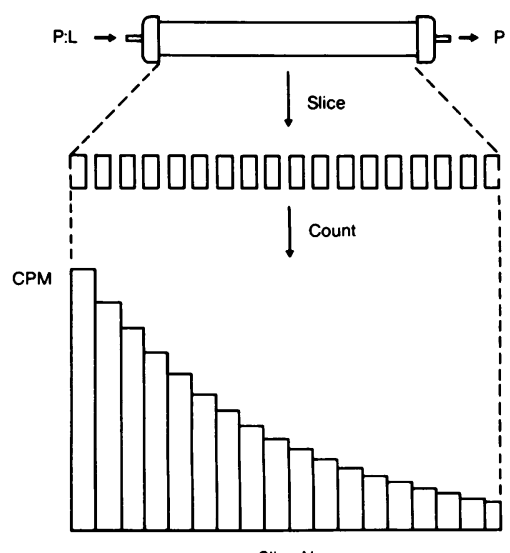

Figure 2. Column slicing method for measuring dissociation. Protein-ligand complex $(P$. $L$ ) containing radiolabeled ligand is rapidly eluted down a column that binds free ligand but not complex. The dissociation rate constant is calculated from the final distribution of oleate on the column. 
dissociation, because otherwise dissociation would not be rate limiting to transfer. For all dissociation events to be detected, rebinding of ligand to albumin from either the free or acceptor-bound pools must be negligible. When these conditions are fulfilled, the amount of ligand transferred to each segment of the column bed is proportional to the amount of complex that reached that segment before dissociating. This amount is determined by slicing the column into segments and measuring the adsorbed ligand (Fig. 2). Since distance along the column bed is proportional to time, the amount of complex remaining undissociated vs. time is readily measured. The dissociation rate constant is then determined by fitting this relationship to a simple exponential curve. The validity of applying this method to the oleate-albumin binding system is discussed in Results.

After equilibration with Krebs-tricine buffer, the Lipidex was carefully packed in $5 \times 100$-mm glass tubes (737-0222; Bio-Rad Laboratories, Richmond, CA) and perfused at about $1.5 \mathrm{ml} \cdot \mathrm{min}^{-1}$ with the same buffer using a precision peristaltic pump (Varioperpex II; LKB Instruments, Sweden). Column temperature was maintained as specified using a thermoregulated water jacket (Isolab, Inc., Akron, $\mathrm{OH}$ ). The transit time of each column (typically $25 \mathrm{~s}$ ) was determined using ${ }^{125} \mathrm{I}$-albumin. In later studies, ${ }^{125}$ I-albumin was replaced by a $6-\mu l$ bolus of $10 \%$ blue dextran, which gave similar results.

In most cases, the $\left[{ }^{3} \mathrm{H}\right]$ oleate-albumin complexes $(100 \mu$ l containing $0.5 \mu \mathrm{M}$ oleate and $1 \mu \mathrm{M}$ albumin) were injected just above the surface of the column bed at $2 \mu \mathrm{l} / \mathrm{s}$ with a Hamilton repeating syringe (Hamilton Co., Reno, NV), and the column eluted for four transit times. The entire column was then cooled to $-70^{\circ} \mathrm{C}$ and placed in a custom made precooled aluminum block with a screw-driven plunger designed to force out the frozen Lipidex core. At regular intervals core segments were sliced free with a razor blade and allowed to fall directly into scintillation vials for determination of radioactivity. Gel extrusion was facilitated by pretreatment of the glass tubes with $5 \%$ dimethyldichlorosilane as previously described (14). Although more cumbersome than the first method, this method allows better time resolution (typically 2 measurements per s $\mathrm{s}^{-1}$ ).

Data analysis. Data for replicate experiments were analyzed separately and the resulting parameters were averaged to determine the mean and standard error values presented in the tables. In vitro data were analyzed by nonlinear least-squares regression analysis using a model consisting of the sum of one or more exponentially decaying pools with or without a constant pool. Of these candidate models, the one selected for representing the data had the lowest sum of squares while still identifying reasonable values for all parameters. Incubation time was measured directly in the albumin-agarose experiments, whereas in the Lipidex experiments it was calculated as the fraction of the column transit time (measured using ${ }^{125}$ I-albumin) required for the albumin complexes to reach each slice. In these analyses the fractional uncertainty of each data point was assumed to be constant and the data weighted accordingly. For calculating the slopes of the Arrhenius curves, measured uncertainties (standard errors) were used instead.

To calculate the apparent rate constant for removal of oleate from the plasma within the sinusoids, the liver was assumed to consist of an array of equivalent parallel sinusoids. According to the sinusoidal (parallel tube) model $(19,20)$, hepatic removal can be represented by the standard equation for exponential decay, Eq. $1: C=C_{0} e^{-k t}$, where $C$ is the oleate concentration in the effluent, $C_{0}$ is the concentration of oleate in the entering plasma, $k$ is the removal rate constant (net fraction of the oleate removed from the plasma at each point within the sinusoids per unit time), and $t$ is the time required for the perfusate to pass through the sinusoids. The relationship of this simple equation to previously defined transport models is presented in the Appendix. Solving Eq. 1 for $k$ gives Eq. 2: $k=-\ln \left(C / C_{0}\right) / t$. The sinusoidal transit time for each liver was calculated from the perfusate flow rate $(Q)$ and volume $(V)$ of the hepatic sinusoidal and Disse' spaces accessible to the albumin according to Eq. 3: $t=V / Q$. The assumptions implicit in the use of these equations will be discussed later.

A value of $0.15 \mathrm{ml} \cdot \mathrm{g}$ liver $^{-1}$ was used for $V$ as previously determined by Blouin and coworkers (21). This value was determined by morphometric analysis of electron micrographs of rat livers that had been per- fusion fixed under conditions very similar to those employed here, and is somewhat smaller than the intrahepatic albumin space estimated by analysis of indicator dilution curves (22). This difference may be explained by the fact that the indicator dilution method measures not only the sinusoidal and Disse spaces, but also hepatic vascular spaces that are not involved in oleate transport.

Apparent activation energies were calculated from the slope of the best fit Arrhenius plot as determined by linear regression. To avoid possible interference by membrane phase transitions occurring at lower temperatures, only data for temperatures between $27^{\circ}$ and $37^{\circ} \mathrm{C}$ were analyzed in the perfused liver experiments.

\section{Results}

Validation of in vitro assays. In the absence of albumin, adsorption of free oleate onto the acceptor beads was rapid in both in vitro systems. For the Lipidex column method, $>99 \%$ of the oleate was adsorbed by the first centimeter of the column after injection of $100 \mu \mathrm{l}$ of freshly prepared $0.5 \mu \mathrm{M}$ oleate at $37^{\circ} \mathrm{C}$ (adsorption rate constant, $>1 \mathrm{~s}^{-1}$ ). For the albumin-agarose method, adsorption of free oleate to the acceptor beads was too rapid to measure. In each experiment a more slowly adsorbing pool of radioactivity was also detected (adsorption rate constant, $0.002-0.015 \mathrm{~s}^{-1}$ ) that accounted for $5-15 \%$ of the total radioactivity added. Increasing the free oleate concentration to $5 \mu \mathrm{M}$ increased this pool to $>40 \%$ of the total, whereas elimination of calcium and magnesium salts from the buffer reduced but did not eliminate it. These results may be explained by formation of aggregates of oleate that adsorb onto the acceptor beads less rapidly than free monomer. Dimerization of fatty acids has been reported at micromolar concentrations (23) and acid soaps may form in the presence of calcium and magnesium (24). Aggregate formation was minimized in the following studies by using only freshly prepared solutions and, in the case of the albumin-agarose experiments, by eliminating calcium and magnesium from the solutions.

In the presence of albumin, adsorption of oleate onto the acceptor beads was much slower $\left(<0.2 \mathrm{~s}^{-1}\right)$ than for free oleate. In both assay systems transfer of oleate from albumin to the acceptor beads was effectively monoexponential, with $>90 \%$ of the total radioactivity typically accounted for by a single exponential process (Fig. 3). As before, a minor pool of radioactivity was noted that adsorbed to the acceptor beads more slowly. This pool is believed to reflect aggregates of oleate and therefore is not considered in the following analysis.

Binding of oleate to each acceptor was avid and effectively irreversible. In both assay systems, $>97 \%$ of the total radioactivity was associated with the acceptor beads at equilibrium. In contrast, no binding of ${ }^{125}$ I-labeled albumin to either Lipidex or albumin-agarose could be detected under standard assay conditions. Oleate did not redistribute on the Lipidex column during a 5-min wash with buffer that included $502-\mu$ injections of 10 $\mu \mathrm{M}$ albumin. These results indicate that the transfer between albumin and the acceptor beads is effectively unidirectional for both methods.

Rate of dissociation in vitro. The apparent rate constant for dissociation of oleate from albumin was $0.14 \mathrm{~s}^{-1}$ (dissociation half-time, $5 \mathrm{~s}$ ) at $37^{\circ} \mathrm{C}$ by both methods (Table I). For this result to reflect the true dissociation rate constant, the albumin concentration must be low enough for rebinding of free oleate to the albumin to be negligible. To determine if this was true, we measured the effect of the albumin concentration on the apparent dissociation rate constant. 


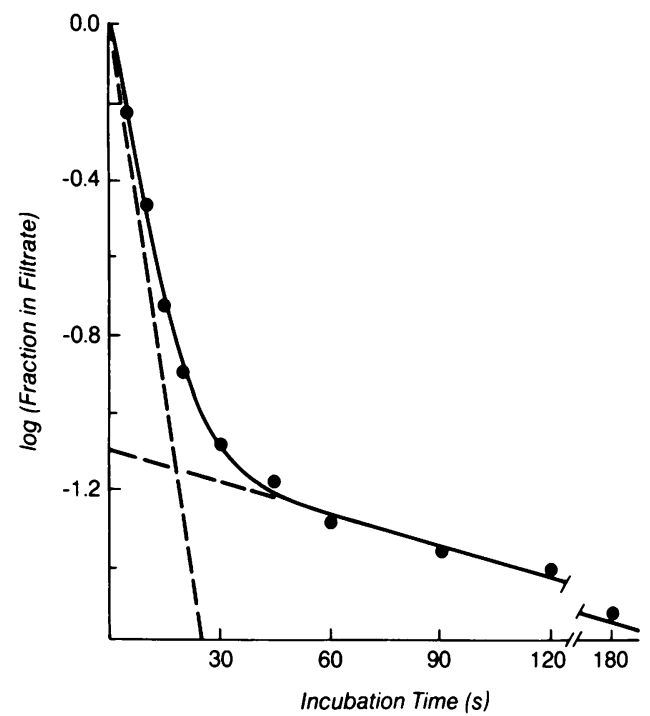

Figure 3. Transfer of oleate from albumin to albumin-agarose at $37^{\circ} \mathrm{C}$. The fraction of the oleate remaining in the soluble phase is shown on a logarithmic scale vs. time. Two components were defined by nonlinear regression analysis (dashed lines). The more rapid component accounted for $>90 \%$ of the total oleate and reflects dissociation of oleate from albumin. The slower component may represent aggregates of oleate including dimers and acid soaps (see text). Results are shown for a typical experiment in which $100 \mu$ l of 1:2 oleate:albumin complex containing $10 \mu \mathrm{M}$ albumin was added to $17 \mathrm{ml}$ of $30 \%$ albumin-agarose gel. Similar results were obtained by the column-slicing method.

As shown in Fig. 4, results were independent of the albumin concentration in the injected solution for all concentrations tested with the albumin-agarose method, and for concentrations below $\sim 2-5 \mu \mathrm{M}$ with the Lipidex method. Extrapolation of the curves for the Lipidex data to zero albumin concentration gave values for the dissociation rate constant similar to those measured directly $\left(0.15 \pm 0.01 \mathrm{~s}^{-1}\right.$ at $37^{\circ} \mathrm{C} ; 0.049 \pm 0.012 \mathrm{~s}^{-1}$ at $23^{\circ} \mathrm{C}$ ).

Temperature sensitivity of dissociation. Dissociation was markedly temperature sensitive. Arrhenius plots of the in vitro

Table I. Rate Constants for Dissociation of Oleate from Albumin

\begin{tabular}{llll}
\hline Temperature & Lipidex & Albumin-agarose & Perfused liver \\
\hline${ }^{\circ} \mathrm{C}$ & $s^{-1}$ & $s^{-1}$ & $s^{-1}$ \\
37 & $0.140 \pm .003(12)$ & $0.142 \pm .017(5)$ & $0.158 \pm .040(5)$ \\
30 & $0.093 \pm .010(4)$ & $0.089 \pm .013(5)$ & $0.086 \pm .019(5)$ \\
23 & $0.049 \pm .012(6)^{*}$ & $0.037 \pm .010(15)^{*}$ & $0.049 \pm .012(5)$ \\
15 & $0.048 \pm .013(4)$ & $0.030 \pm .005(5)^{\ddagger}$ & $0.026 \pm .008(5)^{\ddagger}$
\end{tabular}

Mean \pm SEM (number). Apparent rate constants for dissociation of oleate from bovine albumin in the presence of nonspecific oleate acceptors (Lipidex or albumin-agarose) was compared with the rate of oleate removal from similar solutions by the perfused rat liver. To facilitate comparison, removal is expressed as the fraction of the total oleate within the hepatic sinusoidal space removed by the liver per unit time. Unless otherwise specified, results are mean values for the number of determinations listed in parentheses. * Value from extrapolation of data in Fig. 4 to zero albumin concentration. " From extrapolation of Arrhenius curve to $15^{\circ} \mathrm{C}$.

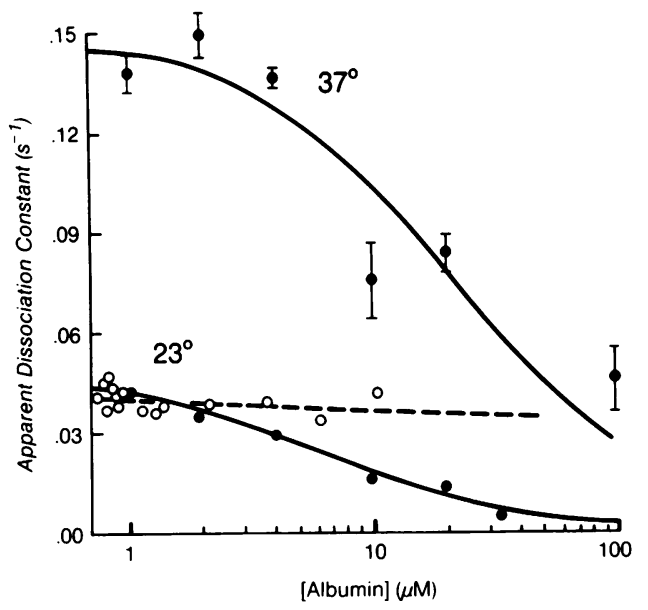

Figure 4. Effect of albumin concentration on dissociation rate constant. Results are shown for Lipidex (solid circles) and albumin-agarose (open circles). Symbols represent individual experiments except where error bars are included. The Lipidex curves are the best fit to a simple model based on Fig. 1 in which higher albumin concentrations reduce the transfer rate by competing with the acceptor beads for free ligand. Each curve is defined by the equation $Y=P 1 /(P 2+\mathrm{X})$, where $Y$ is the apparent dissociation rate constant, $P 1 / P 2$ is the true rate constant, $\mathrm{X}$ is the albumin concentration, and $P 2$ is the albumin concentration at which the rate of rebinding of free oleate to albumin equals the rate of binding to Lipidex. Extrapolation of these curves to zero albumin concentration gave rate constants similar to those determined directly.

dissociation rate constant were linear between 23 and $37^{\circ} \mathrm{C}$ (Fig. 5 , upper portion), although some deviation from linearity was apparent below $23^{\circ} \mathrm{C}$. The apparent activation energy calculated from the linear portion was $12.5 \pm 0.3 \mathrm{kcal} / \mathrm{mol}$ using albuminagarose $(n=5)$ and $10.8 \pm 2.2 \mathrm{kcal} / \mathrm{mol}$ using Lipidex $(n=3)$. This difference was not statistically significant $(P>0.3)$.

Rate of hepatic removal. In five perfusions, the mean sinusoidal transit time calculated using Eq. 2 was $3.41 \pm 0.19 \mathrm{~s}$, based on a perfusate flow rate of $2.66 \pm 0.13 \mathrm{~cm}^{3} \cdot \min ^{-1} \cdot \mathrm{g}^{-1}$. Singlepass extraction of the oleate from a $10-\mu \mathrm{M}$ solution of albumin

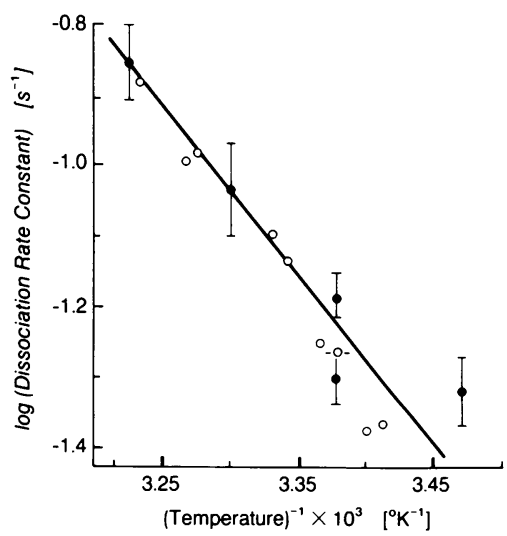

Figure 5. Arrhenius plot of in vitro dissociation rates. Plots were linear between 23 and $37^{\circ} \mathrm{C}$ for both the Lipidex method (solid circles) and the albumin-agarose method (open circles), although some deviation from linearity was seen between 15 and $23^{\circ} \mathrm{C}$ (lower portion of plot). The apparent activation energy of dissociation between 23 and $37^{\circ} \mathrm{C}$ was not different from

the apparent activation energy for oleate uptake by the perfused rat liver. Lipidex points reflect the mean of four to seven determinations. Albumin-agarose data are for individual experiments. For clarity, only a single line has been drawn reflecting the composite data. Lines fit selectively to the Lipidex or albumin-agarose data were not significantly different (see text). 
was $37.8 \pm 0.02 \%$ at $37^{\circ} \mathrm{C}$. Control experiments indicated no measurable extraction of ${ }^{125} \mathrm{I}$-albumin under these conditions. From Eq. 2, the corresponding rate constant for removal of oleate from the sinusoidal compartment is $0.139 \pm 0.007 \mathrm{~s}^{-1}$. A similar result $\left(0.158 \pm 0.040 \mathrm{~s}^{-1}\right)$ was obtained when the rate constant for each liver was calculated separately before averaging (Table I). Neither value is significantly different from the rate constant for dissociation of oleate from albumin determined in vitro ( $P$ $>0.5$ ).

Temperature sensitivity of hepatic removal. Arrhenius plots of the apparent hepatic removal rate constant between 27 and $37^{\circ} \mathrm{C}$ were linear (Fig. 6), with an apparent activation energy of $15.5 \pm 1.6 \mathrm{kcal} \cdot \mathrm{mol}^{-1}(n=5)$. This value is not statistically different from the apparent activation energy of dissociation of oleate from albumin $(P>0.1$ vs. both Lipidex and albuminagarose). Cooling did not appear to damage the liver, because extraction of oleate at $37^{\circ} \mathrm{C}$ was unchanged before and after cooling.

\section{Discussion}

Albumin-receptor model. The hepatic removal of a number of albumin-bound ligands displays a pattern that has been called albumin-receptor kinetics. The primary features of this pattern are a removal rate that depends on the albumin-bound rather than equilibrium-free concentration of the transported ligand and apparent saturation of the removal rate as the concentration of the albumin-ligand complexes is increased. This saturation does not appear to reflect carrier-mediated transport or metabolism of the ligand, because it is not seen unless the albumin concentration is varied together with the ligand. Although originally reported for oleate (1), similar kinetics have since been documented for transport of bilirubin (2), sulfobromophthalein $(B S P)^{1}(2)$, and rose bengal (4) by the isolated perfused rat liver, of BSP and oleate by suspended rat hepatocytes (7), and of fatty acids by the isolated perfused rat heart $(5,6)$.

The transport mechanisms responsible for this pattern remain unknown. The first explanation to be offered was the albumin-receptor model $(1,8)$. This model attributes the dependence on bound ligand to a transient interaction of the albuminligand complex with albumin-binding sites on the liver cell plasma membrane that catalyze the transfer of ligand from albumin to the liver cell. Uptake from the free ligand pool is also permitted by this model (i.e., there is no specific requirement for albumin). However, when the free ligand concentration is sufficiently small due to avid binding by albumin, uptake from the bound ligand pool is quantitatively more important than uptake of free ligand. This model has been supported by the demonstration of saturable binding sites for monomeric albumin on hepatocytes (1), by modeling studies (9), and by the very high efficiency of organic anion uptake despite avid binding to albumin (8), and has been discussed in detail $(8,25)$.

Dissociation-limited model. More recently, an alternative model has been advanced that can account for these observations without the need to postulate an interaction of the complexes with the liver $(10,11)$. The dissociation-limited model retains the traditional assumption that the hepatic removal mechanism acts only on free ligand, but it does not assume equilibrium between bound and free ligand within the sinusoids. When he-

1. Abbreviation used in this paper: BSP, sulfobromophthalein.

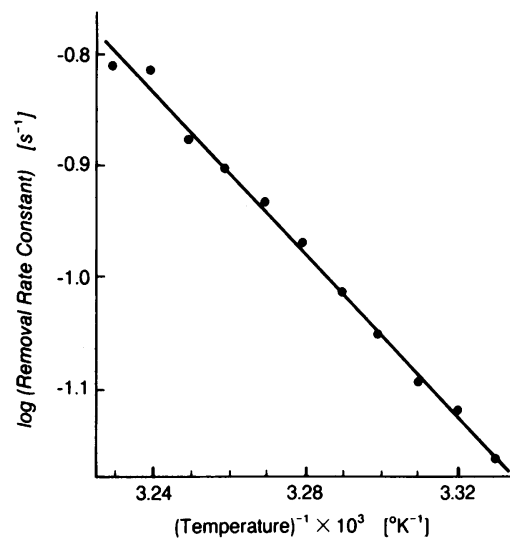

Figure 6. Arrhenius plot of oleate uptake by the perfused rat liver. The apparent rate constant for uptake of oleate from the sinusoidal compartment was determined at steady-state as the liver temperature was varied between 27 and $37^{\circ} \mathrm{C}$. The perfusate contained $10 \mu \mathrm{M}$ albu$\min$ and $1 \mu \mathrm{M}$ $\left[{ }^{3} \mathrm{H}\right]$ oleate in Krebs Henseleit bicarbonate buffer. The apparent activation energy determined from the slope of the best-fit line was $15.5 \pm 1.6 \mathrm{kcal} \cdot \mathrm{mol}^{-1}(n=5)$. Each point reflects the mean of five determinations. Error bars have been omitted for clarity.

patic removal of free ligand from the plasma within the sinusoids is sufficiently efficient, this model predicts that the rate of dissociation of the bound ligand from albumin will determine the removal rate. The dependence of the removal rate on the bound ligand concentration is therefore explained. The apparent saturation seen in increasing the concentration of the complexes is explained by a change in the rate-limiting transport step (from dissociation to influx or metabolism) at higher albumin concentrations, rather than by saturation of a receptor or carrier mechanism.

Because the dissociation-limited model does not assume binding equilibrium, the free ligand concentration within the sinusoids is not a simple function of the concentrations of ligand and albumin. Instead, the free ligand concentration reflects a dynamic balance between processes that consume sinusoidal free ligand (hepatic uptake, binding to albumin, and flow out of the sinusoid) and processes that generate it (efflux from the liver, dissociation from albumin, and flow into the sinusoid) (10). This balance is highly sensitive to the albumin concentration.

In the absence of albumin, removal of efficiently cleared ligands such as fatty acids, taurocholate, and most organic anions is effectively flow-limited (i.e., fractional extractions approach unity) $(1,3,8)$. Addition of albumin reduces the hepatic removal rate by reducing the free ligand concentration. At a sufficiently high albumin concentration, the fractional extraction falls and removal is no longer flow limited. Two possibilities then exist. If dissociation of ligand from albumin is rapid, removal will be limited by intrinsic hepatic transport processes such as influx and elimination. If dissociation is sufficiently slow, however, the rate of removal will be dissociation-limited (10). In this case, still higher albumin concentrations can further reduce the hepatic removal rate until it is slower than dissociation, thereby converting dissociation-limited removal to influx or eliminationlimited removal.

Experiments in which the albumin concentration is varied may therefore involve changes in the transport step that determines the observed removal rate. Failure to fully consider these changes when interpreting kinetic data may lead to incorrect conclusions. The precise conditions under which each transport step is rate limiting and the kinetic behavior expected when the concentration of ligand and/or albumin is varied have been determined (10). These results are fully compatible with the kinetic pattern previously attributed to an albumin receptor. 
Model discrimination. Although the albumin-receptor and dissociation-limited models predict similar kinetics, they differ in the absolute rates of removal predicted. For extensively bound ligands, the removal rate predicted by the dissociation-limited model cannot exceed the rate of spontaneous dissociation from albumin within the hepatic sinusoids, and will equal that rate under dissociation-limited conditions. In contrast, removal may occur more rapidly in the albumin-receptor model due to catalysis of dissociation by the liver.

We found no evidence for catalysis in the current study. Instead, the rate of hepatic oleate uptake from dilute albumin solutions closely paralleled the rate of dissociation of the oleate from albumin within the hepatic sinusoids predicted from in vitro measurements of the dissociation rate constant. These results are in complete accord with the predictions of the dissociation-limited uptake model. They would not be expected for the albumin receptor model unless the increase in uptake efficiency due to catalyzed dissociation were exactly offset by a reduction in efficiency due to other factors. Such a coincidence seems unlikely, particularly since the agreement between the rates of removal and dissociation was seen over a wide range of temperatures (Table I).

As an independent test, we compared the apparent activation energy of the rate-limiting removal step in the perfused liver with that of dissociation in vitro. No differences were seen, suggesting that dissociation is rate-limiting in each case, and that the mechanism of dissociation is not altered by interaction of albumin with the liver. Taken together, these results suggest that the hepatic uptake rate in our study was limited by the rate of dissociation of oleate from albumin within the hepatic sinusoids, and that dissociation of oleate was not catalyzed by a specific interaction of albumin with the liver cell.

This conclusion depends on the validity of four assumptions. First, in comparing in vitro data with data from the perfused liver, we have assumed that dissociation of oleate from albumin is rate limiting in each case. This requires that the rate constants for all other steps be more rapid than dissociation and that rebinding of free oleate to the albumin be negligible (10). These requirements were explicitly confirmed in both in vitro systems. Binding of free oleate to acceptor beads was much more rapid than the observed transfer rate, confirming that the rate constant for adsorption to the acceptor beads was greater than that for dissociation, and the results were largely independent of the albumin concentration used, confirming that the rebinding can be neglected.

Our data suggest that dissociation was also rate limiting in the perfused liver. The single-pass extraction of free oleate by the perfused rat liver under the conditions of the current study averaged 92\% (1). From Eq. 2 the rate constant for removal of free oleate from the sinusoids must have been at least $0.7 \mathrm{~s}^{-1}$, significantly faster than the dissociation rate constant determined in vitro. The albumin concentration selected $(10 \mu \mathrm{M})$ was low enough to allow efficient oleate uptake but was still adequate to bind $>99 \%$ of the oleate in the perfusate (26). Because the hepatic extraction (38\%) was much greater than the unbound fraction of oleate, it is apparent that nearly all fatty acid removed by the liver must have been derived from the bound pool. At such low extractions the removal rate was only minimally limited by plasma flow. We have previously demonstrated that this set of conditions is sufficient to produce dissociation-limited uptake (10).

The second assumption of this study is that the liver can be treated as a collection of equivalent sinusoids. Although sinusoidal heterogeneity is well documented (27), it has little effect on steady-state removal rates unless extraction is high (28). In the current study, the fractional extraction of oleate was always $<40 \%$, suggesting that little error was introduced by using this assumption.

The third assumption is that uptake of ligand within the liver can be described by a single exponential decay process. Eq. 2 treats the entering oleate as a single pool and assumes that the oleate concentration decays exponentially along the length of the sinusoid. Treatment of the oleate as a single pool seems justified because the albumin concentration used was more than 100 times greater than the dissociation constant of the first oleate binding site (26) and molar ratio of oleate to albumin was always $\ll 1$. Under these conditions nearly all the oleate in the perfusate would be expected to be bound to albumin as the 1:1 complex. The assumption of exponential concentration gradients is justified by the demonstration of exponential gradients in our perfusion system for another avidly extracted compound, thyroxine (29). However, even if we had assumed that each sinusoid consisted of a single well-stirred compartment, the rate constant for hepatic removal would have been no more than $25 \%$ larger.

The final assumption is that the rates of oleate uptake and binding to the acceptor beads are first order. Uptake of oleate by the perfused rat liver has been shown to be nonsaturable over the velocity range studied (1). The binding capacity of the acceptor beads always greatly exceeded the amount of oleate bound. Thus, this assumption also seems justified.

The simplest interpretation of these data is that dissociation of oleate from albumin was spontaneous in all cases. However, we cannot exclude the possibility that dissociation was nonspecifically catalyzed to a similar degree by both the liver and in vitro acceptors. Such a result would imply that albumin has the ability to interact efficiently with many different surfaces, including cell membranes, hydrophobic surfaces, and other albumin molecules.

Data exist to support this premise. Thus, the binding affinity of albumin is known to depend on its conformation (30), suggesting that a nonspecific interaction might catalyze dissociation by altering the conformation of the albumin molecule. Moreover, albumin is known to bind to many different cell types (8) and some hydrophobic surfaces (31). Albumin also appears to interact with itself by forming reversible noncovalent dimers at physiologic concentrations $(32,33)$ and with at least one other binding protein, hemopexin, during the transfer of the hydrophobic ligand, heme (34). Similar interactions could conceivably have catalyzed the dissociation of oleate in all three systems studied. In this case the uptake kinetics would still fit the dissociationlimited model, however the rate constants would not be spontaneous but would reflect nonsaturable surface-mediated dissociation as proposed by Forker (3).

Although the dissociation of fatty acids from bovine albumin has not been previously studied, our results agree closely with results for human albumin by Svenson and coworkers (16), who found a rate constant of $0.12 \mathrm{~s}^{-1}$ and an activation energy of 15 $\mathrm{kcal} \cdot \mathrm{mol}^{-1}$ for the dissociation of palmitate. In contrast, significantly slower rate constants (ranging from 0.03 to $0.05 \mathrm{~s}^{-1}$ at $37^{\circ} \mathrm{C}$ ) have been reported for the dissociation of long-chain fatty acids from human albumin by Scheider (35) and Daniels and coworkers (36).

This discrepancy could reflect differences in buffer composition or other experimental details. However, it may be signif- 
icant that in both the current study and that of Svenson, dissociation was monitored by transfer of radiolabeled ligand to an acceptor. In contrast, the slower rate constants were inferred from changes in the dielectric constant or intrinsic fluorescence of the albumin molecule resulting from dissociation. Because the latter methods are sensitive to protein conformation, the slower observed rates might be explained if the change in the conformation of the albumin molecule resulting from dissociation occurred more slowly than dissociation itself. Slow conformational relaxations are known to occur following binding of bilirubin (37) and fatty acids (35) to albumin, and it is reasonable to speculate that similar relaxations may accompany dissociation.

The albumin concentrations used in the current study were selected to minimize rebinding of free oleate, and were therefore much lower than normal plasma levels $(0.4-0.5 \mathrm{mM})$. Because higher albumin concentrations favor binding equilibrium within the hepatic sinusoids (10), dissociation may not be rate-limiting to uptake of oleate under physiologic conditions. Unfortunately, currently available data are insufficient to address this question. Goresky and coworkers (38) have determined the rate constants for palmitate transport in the intact dog. Their results suggest that metabolism should be relatively more important than influx in determining the rate of hepatic palmitate removal at steadystate in vivo. However, the relative importance of dissociation was not addressed by these authors and cannot be inferred from their data.

In conclusion, the liver does not catalyze the dissociation of oleate from albumin more effectively than nonspecific oleate acceptors. Both the rate and temperature sensitivity of dissociation were similar in vitro and in the perfused liver. This conclusion cannot be generalized to other organic anions without more data. Bilirubin, BSP, and rose bengal all bind albumin more avidly and at different sites than oleate (30), and could therefore be handled differently. However, a specific albumin receptor does not appear to participate in the hepatic uptake of oleate.

\section{Appendix}

This study assumes that the hepatic removal of ligand by each sinusoid at steady-state can be represented by the simple Eq. $\mathrm{A} 1: C=C_{0} e^{-k t}$ where $C$ and $C_{0}$ are the total ligand concentrations in the effluent and perfusate, respectively, $k$ is the removal rate constant (net fraction of the ligand removed from the plasma-per-unit time at each point along the sinusoid), and $t$ is the time required for plasma to transit the sinusoid. This is a simplification of an equation derived by Goresky and coworkers in 1973 (39), Eq. A2: $C=C_{0} e^{-\left[k_{1} k_{3} /\left(k_{2}+k_{3}\right)(0 x / W)\right.}$ where $k_{1}$ is the influx rate constant, $k_{2}$ is the efflux rate constant, $k_{3}$ is the sequestration rate constant, $\theta$ is the intracellular-to-plasma space ratio, $x$ is the length of the sinusoid, and $W$ is the flow velocity through the sinusoid.

This equation was developed to study transient uptake events. Under steady-state conditions such as those used in the current study, the unidirectional rate constants $k_{1}, k_{2}$, and $k_{3}$ may be combined into a composite rate constant, $k$, that provides the net rate of removal of total ligand from the plasma (10) as shown in Eq. A3: $k=k_{1} k_{3} /\left(k_{2}+k_{3}\right)$. As previously discussed (10), this expression applies regardless of whether steady-state removal is limited by influx (true when $k_{3} \gg k_{2}$ ), sequestration (true when $k_{2} \gg k_{3}$ ), or a combination of these removal steps (true when $k_{2}$ $\simeq k_{3}$ ). The rate constant $k$ is equivalent to the intrinsic hepatic clearance of total drug as defined by Wilkinson and Shand (40). To facilitate comparison, however, we generally express our rate constants as pools per second (fraction of the relevant pool acted on per unit time, $\mathrm{s}^{-1}$ ) rather than as clearances $\left(\mathrm{ml} \cdot \mathrm{s}^{-1}\right)$. When this is done, the unitless space ratio $\theta$ is unnecessary. Eq. A1 is then obtained by substituting Eq. A3 into Eq. A2 and replacing $\mathrm{x} / W$ with its equivalent, $t$.
The above equations treat plasma ligand as a single compartment. However, ligand exists in the plasma in both bound and unbound compartments that are linked by the rate constants for association $\left(r_{1}\right)$ and dissociation $\left(r_{2}\right)$. These two compartments will behave as a single "plasma" compartment only when binding equilibrium is present. If we make the traditional assumption that uptake is determined by the concentration of unbound ligand, then the rate constant for influx of free ligand by the liver is given by Eq. A4: $k_{1, \text { free }}=k_{1} / \alpha_{3}$ where $\alpha_{3}$ is the unbound fraction of the ligand within the sinusoid. By substitution of $\alpha_{s} k_{1, \text { free }}$ for $k_{1}$ in Eq. A2 and simplifying as before, we can define the rate constant for removal of free drug at steady-state $S$, which is identical to the intrinsic clearance of unbound drug defined by Wilkinson and Shand (40), as seen in Eq. A5: $S=k / \alpha_{s}$. Substituting $\alpha_{s} S$ for $k$ in Eq. Al gives the expression for removal as a function of free ligand, Eq. A6: $C$ $=C_{0} e^{-\alpha_{5} S t}$. When binding equilibrium is present, $\alpha_{3}$ is determined only by the affinity of the binding sites and the concentrations of ligand and albumin within the sinusoid. However, when $S$ is greater than the rate constant for dissociation of bound ligand from albumin, $r_{2}$, free ligand within the sinusoid may be depleted by the uptake process, causing $\alpha_{\mathrm{s}}$ to fall well below the equilibrium value found in the bulk plasma (10). This has the effect of reducing free ligand removal, $\alpha_{s} S$, until it equals ligand dissociation, $r_{2}$. When this occurs, removal is dissociation limited by definition (10).

Substituting $r_{2}$ for $\alpha_{s} S$ in Eq. A6 gives the expression for hepatic removal under dissociation-limited conditions, Eq. A7: $C=C_{0} e^{-r_{2} t}$. Once the criteria for dissociation-limited uptake have been satisfied (see text and reference 10), Eq. A7 may be used to determine the rate constant for dissociation of a given ligand from albumin within a population of uniform hepatic sinusoids.

\section{Acknowledgments}

This work was supported by individual research grant AM-32898 and Liver Center grant AM-26743 from the National Institutes of Health.

\section{References}

1. Weisiger, R., J. Gollan, and R. Ockner. 1981. Receptor for albumin on the liver cell surface may mediate uptake of fatty acids and other albumin-bound substances. Science (Wash. DC). 211:1048-1051.

2. Weisiger, R., J. Gollan, and R. K. Ockner. 1980. An albumin receptor on the liver cell may mediate hepatic uptake of sulfobromophthalein and bilirubin: bound ligand, not free is the major uptake determinant. Gastroenterology. 79:1065. (Abstr.)

3. Forker, E. L., and B. A. Luxon. 1981. Albumin helps mediate removal of taurocholate by rat liver. J. Clin. Invest. 67:1517-1522.

4. Forker, E. L., B. A. Luxon, M. Snell, and W. O. Shurmantine. 1982. Effect of albumin binding on the hepatic transport of rose bengal: surface-mediated dissociation of limited capacity. J. Pharmacol. Exp. Ther. 223:342-347.

5. Hutter, J. F., M. H. Piper, and P. G. Spieckermann. 1984. Myocardial fatty acid oxidation: evidence for an albumin-receptor-mediated uptake process. Basic Res. Cardiol. 79:274-282.

6. Hutter, J. F., H. M. Piper, and P. G. Spieckermann. 1984. Kinetic analysis of myocardial fatty acid oxidation suggesting an albumin receptor mediated uptake process. J. Mol. Cell. Cardiol. 16:219-226.

7. Nunes, R., L. Kiang, and P. D. Berk. 1985. "Albumin receptor" kinetics do not require an intact lobular architecture and are not specific for albumin. Hepatology. 5:1035. (Abstr.)

8. Weisiger, R. A., J. L. Gollan, and R. K. Ockner. 1982. The role of albumin in hepatic uptake processes. Prog. Liver Dis. 7:71-85.

9. Forker, E. L., and B. A. Luxon. 1983. Albumin-mediated transport of rose bengal by perfused rat liver. Kinetics of the reaction at the cell surface. J. Clin. Invest. 72:1764-1771.

10. Weisiger, R. A. 1985. Dissociation from albumin: a potentially rate-limiting step in the clearance of substances by the liver. Proc. Natl. Acad. Sci. USA. 82:1563-1567. 
11. Weisiger, R. A., C. M. Zacks, N. D. Smith, and J. L. Boyer. 1984. Effect of albumin binding on extraction of sulfobromophthalein by perfused elasmobranch liver: evidence for dissociation-limited uptake. $\mathrm{He}$ patology. 4:492-501.

12. March, S. C., I. Parikh, and P. Cuatrecasas. 1974. A simplified method for cyanogen bromide activation of agarose for affinity chromatography. Anal. Biochem. 60:149-152.

13. Greenwood, F., and W. Hunter. 1963. The preparation of ${ }^{131} I-$ labelled human growth hormone of high specific radioactivity. Biochem. J. 89:114-123.

14. Brissot, P., T. L. Wright, W.-L. Ma, and R. A. Weisiger. 1985 Efficient clearance of non-transferrin-bound iron by rat liver: implications for hepatic iron loading in iron overload states. J. Clin. Invest. 76:14631470.

15. Dole, V. 1956. A relation between non-esterified fatty acids in plasma and the metabolism of glucose. J. Clin. Invest. 35:150-154.

16. Svenson, A., E. Holmer, and L.-O. Andersson. 1974. A new method for the measurement of dissociation rates for complexes between small ligands and proteins as applied to the palmitate and bilirubin complexes with serum albumin. Biochim. Biophys. Acta. 342:54-59.

17. Glatz, J. F. C., and J. H. Veerkamp. 1983. A radiochemical procedure for the assay of fatty acid binding by proteins. Anal. Biochem. 132:89-95.

18. Hillier, A. P. 1971. Human thyroxine-binding globulin and thyroxine-binding pre-albumin: dissociation rates. J. Physiol. 217:625-634.

19. Brauer, R. W., G. F. Leong, R. F. McElroy, and R. J. Holloway. 1956. Circulatory pathways in the rat liver as revealed by $\mathrm{P}^{32}$ chromic phosphate colloid uptake in the isolated perfused liver preparation. Am. J. Physiol. 184:593-598.

20. Goresky, C. A. 1963. A linear method for determining liver sinusoidal and extravascular volumes. Am. J. Physiol. 204:626-640.

21. Blouin, A., R. P. Bolender, and E. R. Weibel. 1977. Distribution of organelles and membranes between hepatocytes and nonhepatocytes in the rat liver parenchyma. J. Cell Biol. 72:441-455.

22. Reichen, J., and G. Paumgartner. 1976. Uptake of bile acids by perfused rat liver. Am. J. Physiol. 231:734-742.

23. Mukerjee, P. 1965. Dimerization of anions of long-chain fatty acids in aqueous solutions and the hydrophobic properties of the acids. J. Phys. Chem. 69:2821-2827.

24. Godolphin, W., E. C. Cameron, J. Frohlich, and J. D. Price. 1979. Spurious hypocalcemia in hemodialysis patients after heparinization. In-vitro formation of calcium soaps. Am. J. Clin. Pathol. 71:215218.

25. Ockner, R. K., R. A. Weisiger, and J. L. Gollan. 1983. Hepatic uptake of albumin-bound substances: albumin receptor concept. Am. J. Physiol. 245:G13-G18.
26. Spector, A. A., J. E. Fletcher, and J. D. Ashbrook. 1971. Analysis of long-chain free fatty acid binding to bovine serum albumin by determination of stepwise equibrium constants. Biochemistry. 10:3229-3232.

27. Koo, A., I. Y. S. Liang, and K. K. Cheng. 1975. The terminal hepatic microcirculation in the rat. Q. J. Exp. Physiol. Cogn. Med. Sci. 60:261-266.

28. Bass, L., P. Robinson, and A. J. Bracken. 1978. Hepatic elimination of flowing substrates: the distributed model. J. Theor. Biol. 72: 161-184.

29. Weisiger, R. A., C. M. Mendel, and R. R. Cavalieri. 1986. The hepatic sinusoid is not well stirred: estimation of the degree of sinusoidal mixing by analysis of lobular concentration gradients formed during uptake of thyroxine by the perfused rat liver. J. Pharm. Sci. 75:233-237.

30. Muller, W. E., and U. Wollert. 1979. Human serum albumin as a "silent receptor" for drugs and endogenous substances. Pharmacology. 19:59-67.

31. Paul, L., and C. P. Shorma. 1981. Preferential adsorption of albumin onto a polymer surface-an understanding. J. Coll. Interface Sci. 84:546-549.

32. Mueller, U. W., and J. M. Potter. 1981. Binding of cortisol to human albumin and serum: the effect of protein concentration. Biochem. Pharmacol. 30:727-733.

33. Hartley, R. W., E. A. Peterson, and H. A. Sober. 1962. Relation of free sulfhydryl groups to chromatographic heterogeneity and polymerization of bovine serum albumin. Biochemistry. 1:60-68.

34. Pasternack, R. F., E. J. Gibbs, E. Hoeflin, W. P. Kosar, G. Kubra, C. A. Skowronek, N. M. Wong, and U. Muller-Eberhard. 1983. Hemin binding to serum proteins and the catalysis of interprotein transfer. Biochemistry. 22:1753-1758.

35. Scheider, W. 1979. The rate of access to the organic ligand-binding region of serum albumin is entropy controlled. Proc. Natl. Acad. Sci. USA. 76:2283-2287.

36. Daniels, C., N. Noy, and D. Zakim. 1985. Rates of hydration of fatty acids bound to unilamellar vesicles of phosphatidylcholine or to albumin. Biochemistry. 24:3286-3292.

37. Chen, R. F. 1974. Fluorescence stopped-flow study of relaxation processes in the binding of bilirubin to serum albumins. Arch. Biochem. Biophys. 160:106-112.

38. Goresky, C. A., D. S. Daly, S. Mishkin, and I. M. Arias. 1978. Uptake of labeled palmitate by the intact liver. role of intracellular binding sites. Am. J. Physiol. 234:E542-E553.

39. Goresky, C. A., G. G. Bach, and B. E. Nadeau. 1973. On the uptake of materials by the intact liver. J. Clin. Invest. 52:991-1009.

40. Wilkinson, G. R., and D. G. Shand. 1975. A physiologic approach to hepatic drug clearance. Clin. Pharmacol. Ther. 18:377-390. 